\title{
An Observational Chart Review of a Comparison of Generic to Reference Liposome-Encapsulated Doxorubicin Hydrochloride in Subjects with Epithelial Ovarian Carcinoma Who Have Failed Platinum-Based Chemotherapy
}

\section{Soriano RA* and Gonzalez M}

Envision Clinical Research, LLC, Laredo Texas 78041, USA

${ }^{*}$ Corresponding author: Soriano RA, MSc, Envision Clinical Research, LLC, Laredo Texas 78041, USA, Tel: 9567632216,E-mail: rsoriano@gwu.edu

Citation: Soriano RA, Gonzalez M (2018) An Observational Chart Review of a Comparison of Generic to Reference Liposome-Encapsulated Doxorubicin Hydrochloride in Subjects with Epithelial Ovarian Carcinoma Who Have Failed Platinum-Based Chemotherapy. J Case Rep Stud 6(2): 203. doi: $10.15744 / 2348-9820.6 .203$

Received Date: February 05, 2018 Accepted Date: April 25, 2018 Published Date: April 27, 2018

\begin{abstract}
This study tested for the effects of generic liposome-encapsulated doxorubicin hydrochloride (generic doxorubicin) compared to reference-pegylated liposomal doxorubicin hydrochloride (reference doxorubicin) as a treatment for patients afflicted with epithelial ovarian carcinoma whose disease has progressed after platinum-based chemotherapy. Chart-reviews of actual patients enrolled in a community-based NIH trial suggest that both generic and reference liposomal doxorubicin hydrochloride $(\mathrm{HCl})$ are equally effective as treatment for ovarian cancer carcinoma.
\end{abstract}

Keywords: Doxorubicin; Ovarian Carcinoma; Platinum-Based Chemotherapy

\section{Introduction}

Ovarian Cancer is the $9^{\text {th }}$ most common cancer among women. It ranks $5^{\text {th }}$ in cancer deaths and accounts for more deaths than any other cancer of the female reproductive system [1]. However, in $70 \%$ of cases, the diagnosis is often delayed till the disease is far advanced. Five year relative survival rates show dramatic decreased survival from $71.7 \%$ to $27.4 \%$ in far advanced disease (Figure 1) [2]. These ovarian carcinomas develop mainly in women that are 60 years of age or older [3].

\section{5-Year Relative Survival}

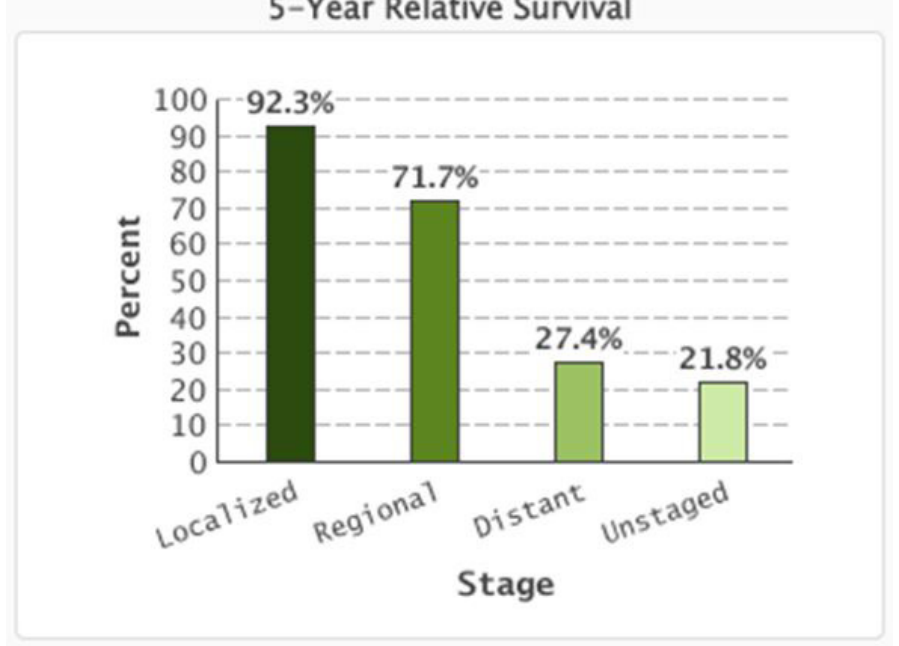

Figure 1: Percent of Cases \& 5-Year Relative Survival by Stage at Diagnosis for Ovarian Cancer

Risk factors include a family history of ovarian cancer, genetic mutations in BRCA1 and BRAC2, Lynch Syndrome, use of estrogenonly hormone replacement therapy, fertility drugs, and obesity. White women have been found to have higher incidence and mortality [4]. 
The initial modality of treatment of epithelial ovarian cancer is a total abdominal hysterectomy and a bilateral salpingooophorectomy with omentectomy. However, if the tumor is found to be well differentiated and in Stage 1A, results from clinical studies with total mortality endpoints supports the first line of chemotherapy with cisplatin, carboplatin (both platinum-based chemotherapy), and paclitaxel (taxane-based chemotherapy). According to the National Cancer Institute of the National Institutes of Health, the incidence of relapse with ovarian epithelial cancer is about $80 \%$ of diagnosed patients that have previously received first-line platinum-based and paclitaxel [4]. The side effects known to be caused (usually after the first cycle) are low blood counts (white cells, red cells and platelets), mouth sores or stomatitis, hand-foot syndrome, arthralgias and myalgias, diarrhea, nausea and vomiting, peripheral neuropathy and hypersensitivity reaction. There is minimal occurrence of neutropenia, nausea, and alopecia. In order to be classified as platinum-refractory or platinum-resistant, subjects need to experience a clinical recurrence within 6 months of completion of a platinum-containing treatment regimen.

Doxorubicin is a chemotherapeutic agent classified as an anthracycline topoisomerase inhibitor (Figure 2) [5]. It was developed in the 1970s against a wide variety of cancers (lymphoma, multiple myeloma, lung, gastric, thyroid, sarcoma, and breast, ovarian and pediatric cancers). There are 2 major mechanisms of antineoplastic activity: 1) intercalation of DNA resulting in inhibition of topoisomerase 11 (TOP2A) and 2) generation of free radicals that lead to cell death. The illustrated diagram shows the pharmacodynamic activity at the nucleus and cell membrane levels that eventually lead to apoptosis or cell death [6,7].

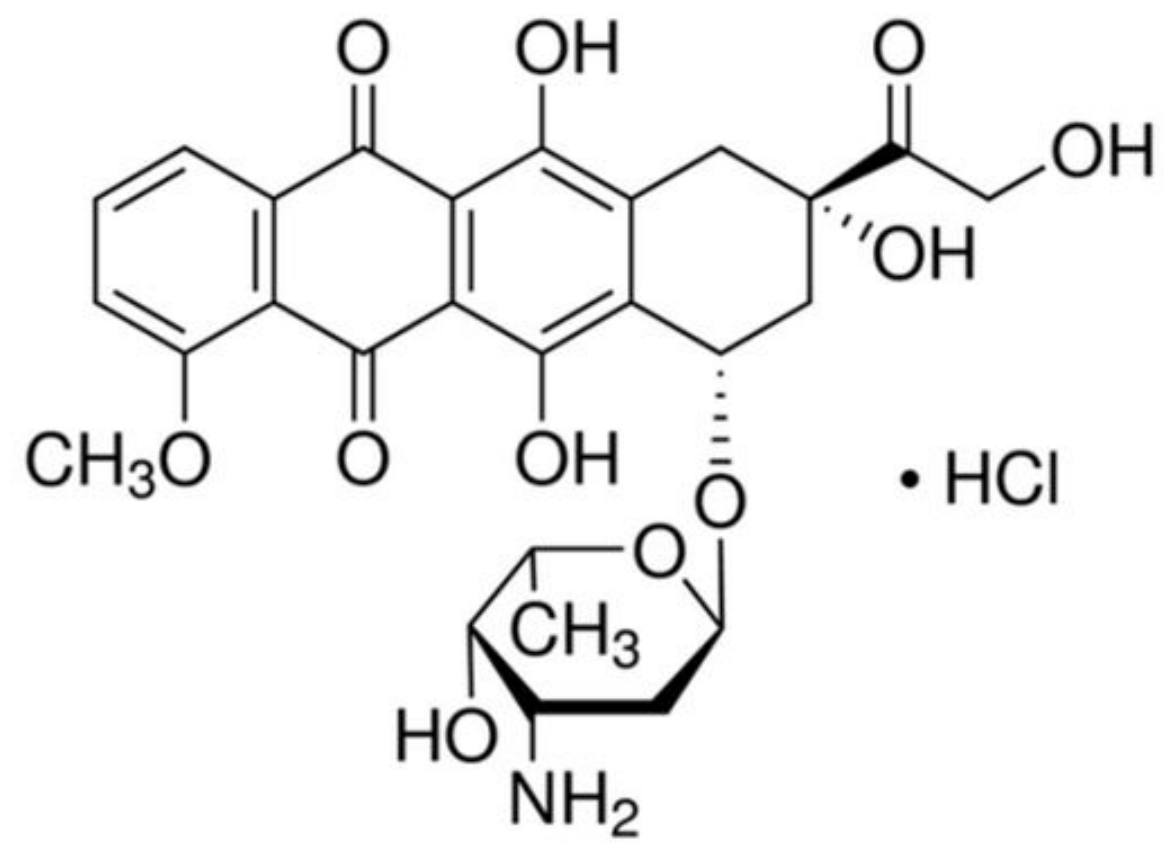

Figure 2: Structural Formula of Doxorubicin $\mathrm{HCl}[5]$

Chemical Formula: Doxorubicin HCl: (8S,10S)-10-[(3-amino-2,3,6-trideoxy-a-L-lyxohexopyranosyl)oxy]-8-glycolyl-7,8,9,10 tetrahydro-6,8,11-trihydroxy-1-methoxy-5,12-naphthacenedione hydrochloride

Empirical Formula: C27H29NO11 $\cdot \mathrm{HCl}$

Molecular Weight: 579.99

Appearance: Red crystalline solid

Below is a diagram, Figure 3, of the Doxorubicin Pathway in a cancer cell adapted from Thorn, Sharon et al. (2010); permission given for reproduction by PharmGKB and Stanford University [7].

Doxorubicin $\mathrm{HCl}$ pegylated liposome injection (reference doxorubicin) has been FDA approved since January 2005 as a treatment for ovarian cancer but the high demand and low supply has resulted in its removal from the pharmaceutical market for 6 years. The purpose of this study was to compare the pharmacokinetic (PK) parameters of reference doxorubicin and generic doxorubicin formulation in subjects with progressive recurrent epithelial ovarian cancer as they affect therapeutic response. Objectives of this observational chart review are to document and quantify the overall efficacy of Generic Doxorubicin in comparison to the Liposome Encapsulated Doxorubicin Hydrochloride. The aim of this chart review was to ascertain on whether the adverse effects of the Generic Doxorubicin were similar in severity as the encapsulated version of the medication. Generic doxorubicin is believed to metabolize in the same manner as reference doxorubicin. Pharmacokinetic monitoring during study trial confirms the bioavailability of the drug as it achieves a mean therapeutic concentration [8]. Other important variables that affect drug absorption, distribution and elimination will need to be considered if there is significant fluctuation in pharmacokinetic distribution in each and amongst subjects. 


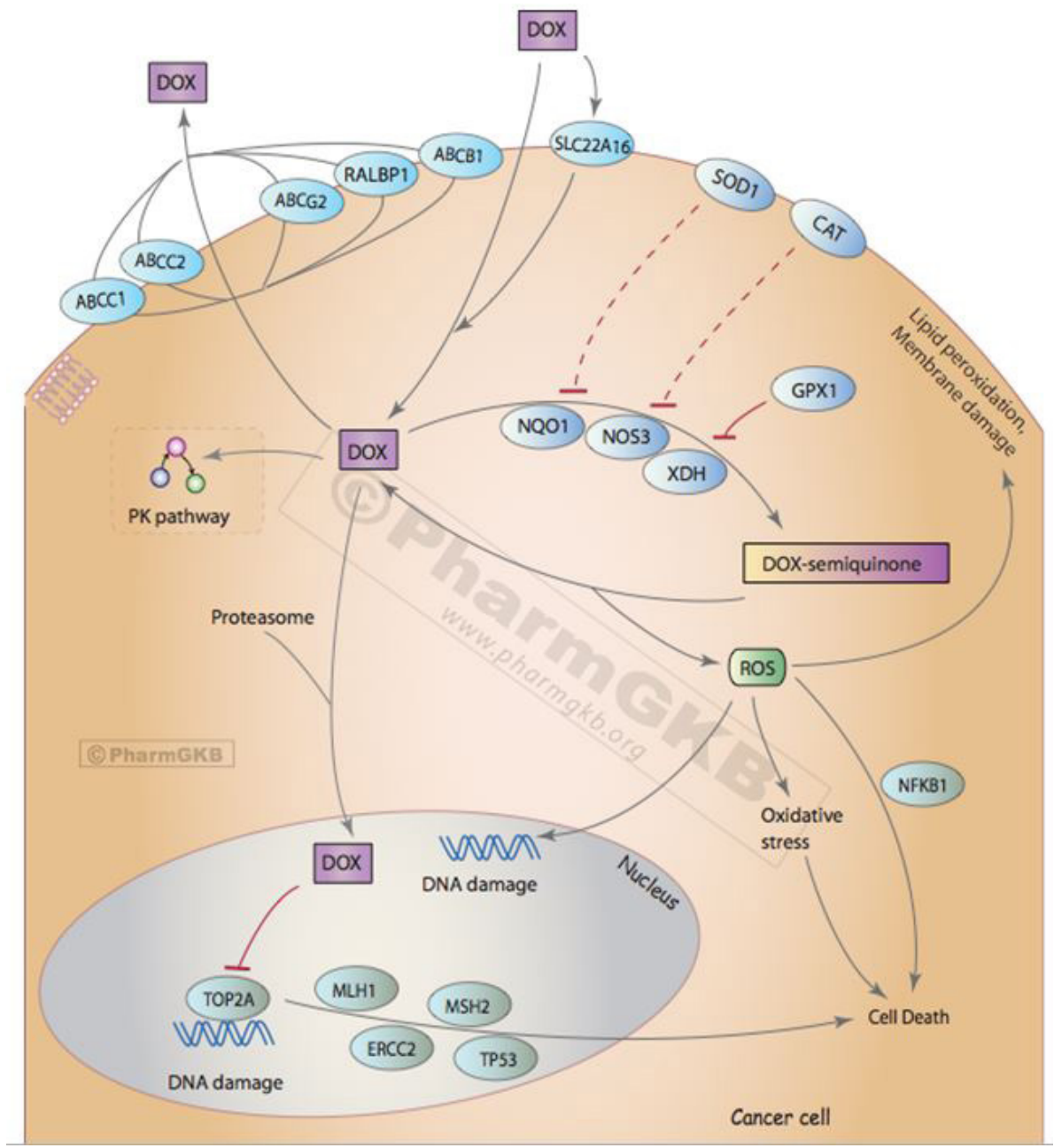

Figure 3: Doxorubicin Pathway in a cancer cell

a)

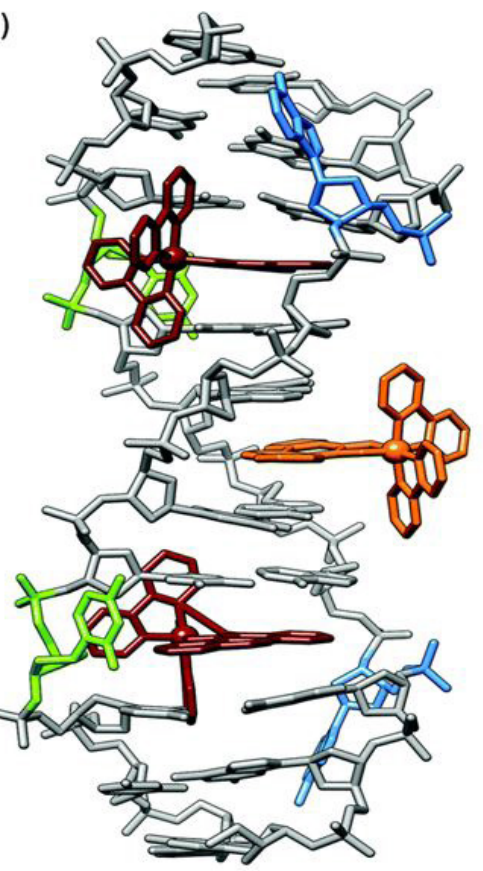

b)

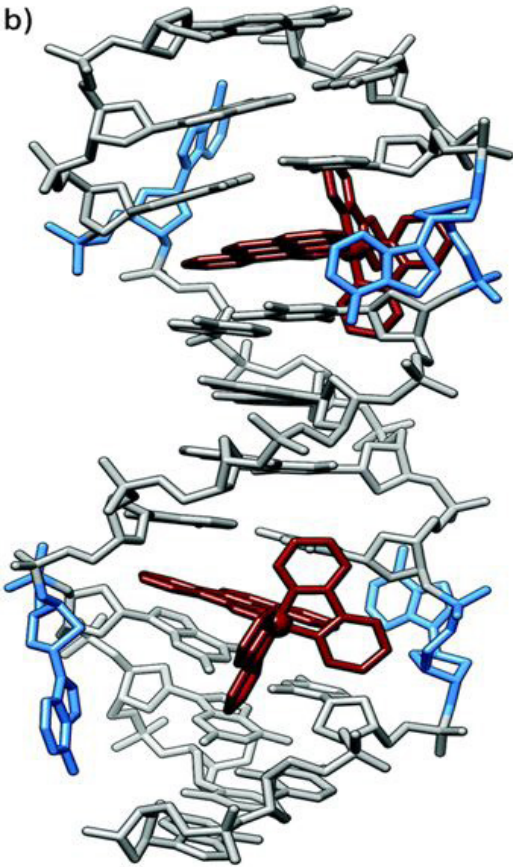

Figure 4: DNA Intercalation of Doxorubicin 


\section{Methodology}

An observational chart review was performed on a community-based NIH multicenter, randomized, single-dose, 2-way crossover, PK bioavailability (BA) study in subjects with epithelial ovarian carcinoma whose disease has progressed or recurred after platinum-based chemotherapy. The total sample size used for the data collection and analysis was inclusive of 5 randomized eligible subjects. Each Subject signed an informed consent for the study participation including PK samples that were necessary for the complete evaluation and analysis of the data. Study subject and bioanalytical laboratory personnel performing data analysis remained blinded with regards to treatment and cohort assignment throughout the trial. Investigators had knowledge of subject treatment and cohort assignment.

The protocol adapted from Gareet [9] was used in this randomized trial. A standardized dose of $50 \mathrm{mg} / \mathrm{m}^{2}$ of either generic doxorubicin or reference doxorubicin were administered as a 1-hour infusion. Each subject was randomized to either drug and received a total of 2 infusions of the same initial drug; the second infusion given 28-42 days or approximately 4 weeks after the first $[9-11]$.

Cancer Antigen 125 (CA-125) is used as a preliminary serologic test to detect ovarian cancer. Cancer Antigen 125 (CA125) was measured on a monthly basis when the patient came in for subsequent treatments. Each subject participated in the study for approximately 12-14 weeks. The subjects had their blood samples for the serologic CA-125 marker 4 times each throughout the course of their participation. Total duration of the study was dependent on the number of days needed to conduct all screening procedures and the presentation of any adverse effects that may delay the initiation of Cycle 2 dosing. The end of the study is marked by the last follow-up visit for the last subject to complete the study period.

All subjects met all inclusion criteria and none of the exclusion criteria, the most notable exclusion criteria being that the patient could not have suffered from another malignancy within the last 5 years. Throughout the duration of the study, patients maintained compliance with study protocol. The subjects had a positive, satisfying quality of life with a great sense of well-being. Screening tests were conducted for baseline vital signs, health parameters such as weight and body mass index, and interval illnesses or medications. The patients' subjective response to the drug was monitored. PK blood levels were monitored to ensure adequate pharmacodynamic response to administered drug dosage (Table 1).

\begin{tabular}{|c|c|}
\hline Inclusion Criteria & Exclusion Criteria \\
\hline $\begin{array}{l}\text { 1. Females between } 18 \text { and } 75 \text { years of age, } \\
\text { inclusive } \\
\text { 2. Histologically proven epithelial ovarian } \\
\text { cancer } \\
\text { 3. Documented progressive or recurrent } \\
\text { disease after treatment with platinum-based } \\
\text { chemotherapy } \\
\text { 4. Clinical Trial by NIH } \\
\text { 5. Life expectancy of at least } 180 \text { days }\end{array}$ & $\begin{array}{l}\text { 1. History of major cardiac, liver, or kidney disease } \\
\text { 2. Active opportunistic infection with mycobacteria, } \\
\text { cytomegalovirus, toxoplasma, Pneumocystis carinii, or other } \\
\text { microorganism. } \\
\text { 3. Severe or life-threatening infection, including known past } \\
\text { medical history of human immunodeficiency virus/acquired } \\
\text { immune deficiency syndrome (HIV/AIDS). } \\
\text { 4. History of other malignancies in the last } 5 \text { years. Subjects } \\
\text { with prior history of in situ cancer or basal or squamous cell } \\
\text { skin cancer are eligible. Subjects with other malignancies that } \\
\text { were cured with definitive primary therapy alone (e.g. surgery) } \\
\text { and who have been continuously free of the previous disease for } \\
\text { 1 year are also eligible upon Sponsor approval. } \\
\text { 5. Pregnant or breast feeding } \\
\text { 6. Use of protocol-specified prohibited medications. } \\
\text { 7. Has exceeded or will exceed a lifetime cumulative dosage } \\
\text { of greater than } 550 \text { mg/m², including receipt of the first } \\
\text { infusion during this study. Prior use of other anthracyclines or } \\
\text { anthracenediones should be included in calculations of total } \\
\text { lifetime cumulative dose. } \\
\text { 8. Evidence of cardiac toxicity History of hypersensitivity } \\
\text { reaction to a conventional formulation of doxorubicin HCl, or } \\
\text { the components of reference doxorubicin. } \\
\text { 9. Pregnant or breast feeding } \\
\text { 10. No longer qualified to receive liposomal doxorubicin } \\
\text { (including but not limited to AEs from prior administration or } \\
\text { disease progression since prior administration) }\end{array}$ \\
\hline
\end{tabular}

Table 1: Inclusion and Exclusion Criteria

\section{Results}

The subject population included 5 women between the ages of 40- 74 and were of White Race and of Hispanic ethnicity. Majority of the patient subjects tolerated treatments well and did not display any signs of rejection against infusions. For a total of 3 subjects, CA125 levels decreased, with the initial levels ranging from 121-1717 and final levels down to 71-324. There was no difference between Cohort 1 and 2. The CA125 levels in two subjects did not change and remained in normal range throughout the study, suggesting that reference doxorubicin helped them remain in remission. There were minor infusion-related side effects several days after such as generalized rash, hyperpigmentation on the extremities, stomatitis, and arthralgia that were self-limited or 
resolved after topical over-the-counter medications (Table 2).

\begin{tabular}{|c|c|c|c|c|c|}
\hline & $\begin{array}{c}\text { Subject 1 } \\
\text { Reference } \\
\text { doxorubicin }\end{array}$ & $\begin{array}{c}\text { Subject 2 } \\
\text { Generic } \\
\text { doxorubicin }\end{array}$ & $\begin{array}{c}\text { Subject 3 } \\
\text { Generic } \\
\text { doxorubicin }\end{array}$ & $\begin{array}{c}\text { Subject 4 } \\
\text { Reference } \\
\text { doxorubicin }\end{array}$ & $\begin{array}{c}\text { Subject 5 } \\
\text { Generic } \\
\text { doxorubicin }\end{array}$ \\
\hline $\begin{array}{c}\text { Initial CA125 } \\
\text { Levels }\end{array}$ & 129 & 121 & 9 & 1717 & 6 \\
\hline $\begin{array}{c}\text { Final CA125 } \\
\text { Levels }\end{array}$ & 97 & 71 & 9 & 324 & 8 \\
\hline
\end{tabular}

Table 2: Initial and Final CA125 levels for subjects randomized for reference doxorubicin and generic doxorubicin

At the conclusion of treatment, subjects all reported that they were feeling very good. The objective decrease in CA125 levels and the subjective feeling of generalized well-being after generic doxorubicin treatment have shown it to be an effective treatment against ovarian carcinoma.

\section{Discussion}

The result of this study supports previous studies on the efficacy of generic liposome-encapsulated doxorubicin hydrochloride in epithelial ovarian carcinoma [9,12-15]. In one study it was demonstrated that median progression-free survival was 5.7 months with an overall survival of 1.5 to $>24$ months [15]. Treatment was well tolerated. Moreover, the study concluded that doxorubicin had substantial activity against ovarian cancer compared to platinum and paclitaxel treatments. In addition, the responses to the doxorubicin treatment were maintained with minimal toxicity [9]. In another study it was proven that treatment with pegylated liposomal doxorubicin significantly prolongs survival compared with topotecan (topoisomerase 1 inhibitor) and platinumsensitive disease in patients with refractory epithelial ovarian cancer [13].

In a study comparing several platinum-based treatment therapies and topotecan, it was found that there was a comparable efficacy amongst them. However, liposomal doxorubicin has a favorable safety profile and a convenient dosing system that makes it a valuable treatment option in subjects with ovarian cancer. It has been studied that the overall survival of subjects treated with liposomal doxorubicin is 108 weeks versus a median survival of 71.1 weeks for subjects treated with topotecan [14].

The efficacy of liposomal doxorubicin may be increased when combined with other treatments. A combination of carboplatin with pegylated liposomal doxorubicin provided superior progression-free survival compared to the combination of carboplatin and paclitaxel [12]. Moreover, the occurrence of less adverse effects associated with pegylated liposomal doxorubicin, in particular lesser risk of cardiomyopathy, makes it a favorable treatment option.

\section{Future Direction}

For future studies, greater validity and a broader range of conclusions may be achieved with large multi-center community-based randomized double blind clinical trials. Moreover, screening for genetic mutations, hereditary diseases and other risk factors may help differentiate effective treatment protocols. The detection of other cancer genetic markers aside from CA125 will define earlier targeted therapies and hopefully improve survival rates.

\section{Conclusion}

The generic doxorubicin formulation is a suitable, comparable and effective alternative to the reference doxorubicin formulation in the treatment of epithelial ovarian carcinoma in patients with epithelial ovarian carcinoma who have failed platinum-based chemotherapy. This is proven by decreased and sustained reduction in CA125 levels and subjects' documented symptomatology reduction in subjects 2,3 , and 5 .

\section{Acknowledgment}

Research facility provided by Envision Clinical Research, 6801 McPherson Road Suite 217 Laredo Texas 78041.

\section{References}

1. Cancer (2014) Key Statistics for Ovarian Cancer, USA.

2. SEER (2014) Cancer Statistics Factsheets: Ovary Cancer, National Cancer Institute, Bethesda, MD, USA.

3. Cancer (2014) Ovarian Epithelial, Fallopian Tube, and Primary Peritoneal Cancer Treatment (PDQ $\left.{ }^{\circ}\right)-P^{2}$ atient Version, USA.

4. National Cancer Institute (2014) Recurrent or Persistent Ovarian Epithelial, Fallopian Tube, and Primary Peritoneal Cancer Treatment, USA.

5. Doxil $^{\oplus}$ (2014) ALZA Corporation Bedford, OH, USA.

6. National Center for Biotechnology Information, USA.

7. Schmidt C (2011) CA-125: A Biomarker Put to the Test. J Natl Cancer Inst 103: 1290-1.

8. Thorn CF, Oshiro C, Marsh S, Hernandez-Boussard T, McLeod H, et al. (2011) Doxorubicin pathways: pharmacodynamics and adverse effects. Pharmacogenet Genomics 21: 440-6. 
9. Gareet S (1989) A Single Dose Pharmacokinetic and Bioavailability Study of a Generic Liposome-encapsulated Doxorubicin Hydrochloride Formulation in Subjects with Epithelial Ovarian Carcinoma Who Have Failed Platinum-Based Chemotherapy. TOLMAR, Inc., USA.

10. Dayton Physicians Network (2013) A Single Dose Pharmacokinetic and Bioavailability Study of a Generic Liposome-Encapsulated Doxorubicin Hydrochloride Formulation in Subjects with Epithelial Ovarian Carcinoma Who Have Failed Platinum-Based Chemotherapy, USA.

11. Louisiana Hematology Oncology Associates (2018) An Uncontrolled Observational Study Providing Continued Access to Care of a Generic LiposomeEncapsulated Doxorubicin Hydrochloride Formulation in Subjects with Epithelial Ovarian Carcinoma who Have Failed platinum-Based Chemotherapy, USA.

12. Gibson JM, Alzghari S, Ahn C, Trantham H, La-Beck NM (2013) The role of pegylated liposomal doxorubicin in ovarian cancer: a meta-analysis of randomized clinical trials. Oncologist 18: 1022-31.

13. Gordon AN, Tonda M, Sun S, Rackoff W, Doxil Study 30-49 Investigators, et al. (2004) Long-term survival advantage for women treated with pegylated liposomal doxorubicin compared with topotecan in a phase 3 randomized study of recurrent and refractory epithelial ovarian cancer. Gynecol Oncol 95 : 1-8.

14. Gordon AN, Fleagle JT, Guthrie D, Parkin DE, Gore ME, et al. (2001) Recurrent Epithelial Ovarian Carcinoma: A Randomized Phase III Study of Pegylated Liposomal Doxorubicin Versus Topotecan. J Clin Oncol 19: 3312-22.

15. Muggia FM, Hainsworth JD, Jeffers S, Miller P, Groshen S, et al. (1997) Phase II study of liposomal doxorubicin in refractory ovarian cancer: antitumor activity and toxicity modification by liposomal encapsulation. J Clin Oncol 15: 987-93.

16. US Biological Life Sciences, USA.

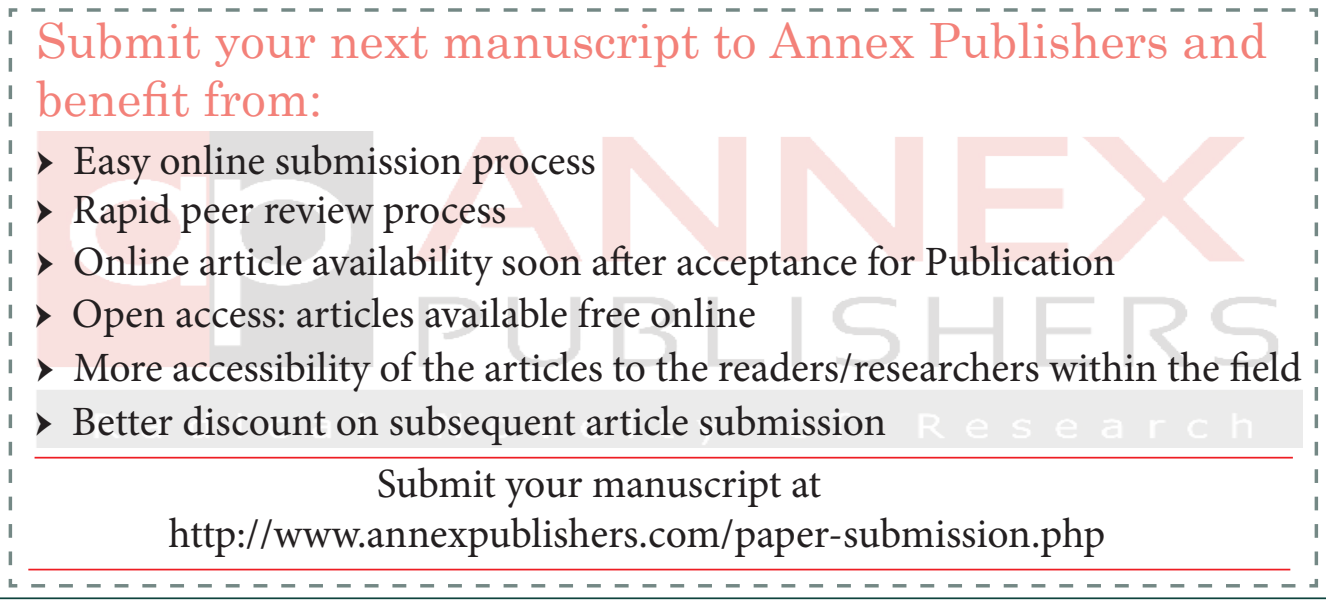

